\title{
Sibling Resemblance for Psychiatric Disorders in Offspring at High and Low Risk for Depression
}

\author{
Richard Rende*†, Priya Wickramaratne*†, Virginia Warner† \\ and Myrna M. Weissman*†
}

\begin{abstract}
This study examined sibling resemblance for major depressive disorder, anxiety disorder, and conduct disorder, in offspring at high and low risk for depression by virtue of parental diagnosis. The sample, which ranged in age from 6 to 23 years, included 164 sibling pairs at high risk, and 68 sibling pairs at low risk. Each cohort was assessed at two waves separated by a 2-year period. Sibling resemblance in the high risk cohort was substantially greater than resemblance in the low risk cohort for anxiety disorder (and comorbid conditions including anxiety disorder), but not depression. Discussion focused on the possibility that anxiety disorder may reflect the most pronounced familial influences common to siblings at high risk for depression.
\end{abstract}

Keywords: Depression, risk, siblings, psychiatric disorders

\section{Introduction}

It is now well-established that offspring of depressed parents are at increased risk for depression, as well as other psychiatric disorders, as compared to offspring of parents with no history of depression. Weissman et al. (1987) reported that offspring of depressed parents had higher rates of major depression and anxiety disorders than offspring of parents with no psychiatric disorders; the offspring at high risk also had higher 2-year incidence rates of major depression and anxiety disorders as compared to the offspring at low risk (Warner et al., 1992). Hammen et al. (1987) found that offspring of parents with affective disorder had increased rates of major depression and conduct disorder as compared to offspring of parents with no psychiatric disorder; Hammen et al. (1990) have also shown that the offspring at high risk had elevated rates of major depression, and anxiety and conduct disorders

\section{Accepted manuscript received 26 April 1995}

*Department of Psychiatry, Columbia University College of Physicians and Surgeons. †Division of Clinical and Genetic Epidemiology, New York State Psychiatric Institute.

Requests for reprints to: R. Rende, Department of Psychology, Busch Campus, Rutgers University, New Brunswick, NJ 08903, U.S.A. 
over a 3-year period as compared to the offspring at low risk. These studies have demonstrated the substantial risk for psychiatric disorders in offspring being reared by parents with affective disorders.

In all the studies conducted to date, the analysis of aggregate rates of disorders has been conducted as if the observations are independent of one another. However, as more than one observation per family may be made (i.e. more than one child per family may be studied), the observed data on high risk offspring may contain dependent data. Although the dependence of observations has been regarded as a fundamental methodological problem in the calculation of familial aggregation (see Weissman et al., 1986), statistical methods which overtly assess the degree of similarity between dependent observations such as siblings may provide insight on the way in which psychopathology aggregates within families (Rende \& Weissman, in press), as described below.

Table 1. Hypothetical patterns of sibling resemblance in offspring at high risk for depression

\begin{tabular}{|c|c|c|}
\hline \multicolumn{3}{|c|}{ Example 1} \\
\hline $\begin{array}{l}1 \\
2 \\
3 \\
4\end{array}$ & $\begin{array}{c}\text { Depressed } \\
\text { Depressed } \\
\text { Not depressed } \\
\text { Not depressed } \\
\\
\text { e of affected offs }\end{array}$ & $\begin{array}{c}\text { Not depressed } \\
\text { Not depressed } \\
\text { Depressed } \\
\text { Depressed }\end{array}$ \\
\hline Family & $\begin{array}{c}\text { Example } 2 \\
\text { Sibling } 1\end{array}$ & Sibling 2 \\
\hline $\begin{array}{l}1 \\
2 \\
3 \\
4\end{array}$ & $\begin{array}{c}\text { Depressed } \\
\text { Depressed } \\
\text { Not depressed } \\
\text { Not depressed }\end{array}$ & $\begin{array}{c}\text { Depressed } \\
\text { Depressed } \\
\text { Not depressed } \\
\text { Not depressed }\end{array}$ \\
\hline
\end{tabular}

Aggregate rate of affected offspring $=50 \%$

Before discussing specific methods, consider the hypothetical patterns of familial aggregation presented in Table 1. In example 1, eight offspring of depressed parents are assessed for major depressive disorder; these eight offspring represent four families, each of which contribute two offspring to the study. In this example, four of the eight offspring (50\%) are diagnosed as having a lifetime history of major depression. In example 2, an identical design is used, and again the aggregate rate of depression in the sample is $50 \%$. Note, however, that in example 1, all sibling pairs have a different outcome, whereas in example 2, all sibling pairs have the same outcome. Even though the aggregate rate of depression in offspring is identical in the two examples, opposite patterns of sibling resemblance exist, each of which carries very different implications for the etiology of depression in offspring at risk. Hence, using statistical methods which assess associations between dependent 
observations may reveal patterns of resemblance which cannot be discerned from aggregate rates of disorders.

The need for a specific focus on sibling resemblance in studies of offspring at high risk is highlighted by recent interest in differentiating between shared and nonshared influences on siblings. Traditional theories of the family suggested that siblings would have similar outcomes because they share rearing environments provided by parents as well as shared genetic backgrounds. In this sense, siblings would experience shared environmental influences in that their rearing environment was common. Hence, with respect to the offspring of depressed parents, it would be assumed that siblings with a depressed parent would be at equal "risk" to develop depression, for both genetic and environmental reasons.

Although this approach makes intuitive sense, over the last decade a number of studies have suggested that siblings often have very different outcomes in many domains of development. Two reasons for differences between siblings should be considered. A first point is that as siblings share on average only $50 \%$ of additive genes, genetic differences between siblings may lead to differential outcomes for siblings. A second point is that behavioral genetic studies have provided little evidence that siblings share common environmental influences within the family. Because of this, it has been suggested that siblings experience very different "environments" growing up, called nonshared environmental influences, which operate to produce dissimilarities among siblings (see Dunn \& Plomin, 1990). For example, although there are specific styles of parenting (e.g., low warmth, high conflict) which place offspring at risk for depression, siblings of a depressed parent may not necessarily be exposed, to the same degree, to these styles (Rende \& Plomin, 1993; Rutter, 1990). A recent study found that siblings at high risk for depression do in fact show low correlations for typical measures of the family environment, which suggests that the "risk" for psychiatric disorders may not be shared equally by siblings (Wickramaratne et al., submitted). The emerging data documenting nonshared influences on siblings suggest that empirical methods are necessary to determine the extent to which siblings at risk resemble each other in terms of psychopathology (Reiss, Plomin \& Hetherington, 1991; Rende \& Plomin, 1993), in order to understand the precise ways in which parental psychopathology places children at risk. However, to date empirical studies of sibling resemblance for depression and other psychiatric disorders have not yet been conducted (Rutter et al., 1990).

The purpose of this study is to examine sibling resemblance in a longitudinal study (e.g. Warner et al., 1992; Weissman et al., 1987) of offspring at high and low risk for depression by virtue of parental diagnosis. Sibling resemblance is assessed for three disorders-major depressive disorder, anxiety disorder, and conduct disorder-because of the evidence reviewed earlier that offspring of depressed parents have relatively high rates of these disorders. Sibling resemblance is assessed for the first wave of the study (in which offspring were between 6 and 23 years of age) and a 2-year follow-up of the original sample. Sibling resemblance is indexed using pairwise odds ratios, a nonparametric statistic which can accommodate variable numbers of siblings within a family (Khoury, Beaty \& Cohen, 1993); details of the analytic approach are outlined in the Methods section. In addition, because 
of the high rates of comorbidity found for disorders in childhood and adolescence (e.g. Caron \& Rutter, 1991), we also examine sibling resemblance for comorbid conditions (i.e. anxiety and depression, anxiety and conduct disorder, depression and conduct disorder).

A first general hypothesis is that sibling resemblance for psychiatric disorders in the high risk sample will be significant. We expect sibling resemblance for major depression and anxiety disorder because of either common genetic or environmental influences derived from having a depressed parent; sibling resemblance for conduct disorder is expected because nonspecific family factors related to parental depression, such as family discord, may also be risk factors for externalizing problem behavior (e.g. Rutter, 1990). A second hypothesis is that sibling resemblance for comorbid conditions, especially depression and anxiety disorder, will be significant in the high risk sample. We expect sibling resemblance for comorbidity because of the evidence that depression and anxiety may be due to common genetic influences (Kendler et al., 1992). A third hypothesis is that sibling resemblance for these disorders will increase after the second timepoint of the study, as more offspring pais through the age of first onset for these disorders. A fourth hypothesis is that sibling resemblance for the three disorders of interest will not be significant in the low risk group, given the absence of parental psychopathology and the general finding that sibling resemblance for low risk groups is low for many domains of development (Dunn \& Plomin, 1990).

\section{Method}

\section{Sample}

This study is based on 220 children at high and low risk for major depression by virtue of the presence or absence of major depression in their parents (as defined by Research Diagnostic Criteria). A complete description of the parents and their assessments has been described elsewhere (Weissman et al., 1987). Previous reports from this study have compared 125 children of 56 depressed proband parents with 95 children of 35 normal proband parents. In psychiatric assessments of probands and their spouses upon reinterview three normal probands reported a lifetime history of major depression; thus, the probands were restratified by diagnosis. The final group included 220 offspring from 91 families who were between the ages of 6 and 23 years at initial interview (Wave 1), with 153 offspring from 65 families with one or more parent depressed. Diagnostic information from parents and/or offspring was available on all 220 offspring at Wave 1. Offspring were on average 16.7 years of age; approximately $53 \%$ of the offspring are female. Offspring at high and low risk were matched in terms of age, gender, social class, age of parents, and religion. All subjects in the study were Caucasian.

Two years after the initial interview, all 91 families were recontacted. Eighty-five (93\%) of the 91 families consented to participate in the second interview. One hundred and seventy-four offspring had diagnostic information from the second interview (with either parent or offspring) and 46 had information only from the initial interview. Comparisons between these 46 subjects and the other 174 offspring found no significant differences on sex, parental diagnoses, offspring's diagnoses, marital adjustment, divorce status, child's temperament and comorbidity in the offspring; the 46 subjects tended to come from lower social classes. The mean age of offspring at Wave 2 was 18.5 years of age (range 6-28).

In this report analyses are based on the subsample of families which contributed more than one offspring to the study. Forty-four $(67.7 \%)$ of the families in the high risk sample, and $23(88.5 \%)$ of the families in the low risk sample, contributed more than one offspring. In the high risk sample, a 
total of 164 sibling pairs (45 male-male, 39 female-female, 80 male-female) were available for analyses, 68 pairs of siblings (16 male-male, 21 female-female, 31 male-female) were available in the low risk sample.

\section{Assessment of offspring}

A modified version of the Schedule for Affective Disorders and Schizophrenia for School-Aged Children, Epidemiological Version (Orvaschel et al., 1981) formed the core of a comprehensive interview administered to the parent about the child and to the child about him or herself. Interviewers, who were unaware of the parents' diagnosis, interviewed a parent (usually the mother) about the child, and at a later date interviewed the child.

\section{Diagnosis in offspring}

In the present analysis, only diagnoses of major depression, anxiety disorder, and conduct disorder were used. Strict criteria were used to make the diagnosis of a lifetime episode of Major Depression: a duration of at least four weeks and an impairment in a major social role. DSM-III criteria were used for the lifetime diagnosis of conduct disorder and anxiety disorder. Diagnoses were only assigned if a subject met "definite" diagnostic criteria. Diagnoses in offspring were made according to a best estimate procedure (Leckman et al., 1982), in which a child psychiatrist and psychologist, who were not involved in the interviewing, reviewed all sources of information and blindly assigned a lifetime diagnosis based on the Diagnostic and Statistical Manual of Mental Disorders, 3rd edition (American Psychiatric Association, 1987). Discrepancies in diagnoses by the independent evaluators were resolved by a third source, who also independently and blindly reviewed all information. In an attempt to assess the reliability of the best estimate procedure, a second child psychiatrist reviewed all available information on 38 randomly selected children and made best estimate diagnoses. Agreement between psychiatrists on children's diagnoses was excellent (e.g. kappa coefficient $=0.89$ for major depression, and $=0.93$ for conduct disorder). Rates of these disorders in the offspring at high and low risk at Waves 1 and 2 have been published elsewhere (Weissman et al., 1987; Warner et al., 1992).

\section{Statistical analysis}

Sibling resemblance for each disorder was determined using the pairwise odds ratio, which is analogous to the intraclass correlation coefficient for a dichotomous variable. The pairwise odds ratio for any two siblings is defined as follows: it is the ratio of the odds that, given that any sibling selected at random (from the set of siblings within a family) has the disorder, a second sibling selected at random will also have the disorder, over the odds that, given that any sibling selected at random does not have the disorder, a second sibling picked at random will have the disorder. These odds ratios are computed using all possible pairs of siblings within a family (Hunt et al., 1986; Khoury et al., 1993); e.g. a sibship of 3 would contribute 3 unique pairs. Such "multiple counting" of individuals does not produce biased test statistics, because ". . . if sibs are truly independent of one another in risk, it will not matter if any one sib appeared in several pairs when constructing the final table" (Khoury et al., 1993, p. 190). This property of the pairwise odds ratio makes it a more suitable analytic technique than other nonparametric indices such as concordance, which assume only two observations per family as in the case of twins (see McGue, 1991). An odds ratio of one indicates that the diagnostic status of one sibling is independent of the diagnostic status of the other sibling; odds ratios greater than one indicate sibling resemblance. The significance of the odds ratio is determined using the $\chi^{2}$ statistic. A formula for calculating the pairwise odds ratio is as follows, based on categorizing each sibling pair into concordant (both sibs affected) and discordant (one sib affected) pairs: If the number of concordant pairs is $X$, the number of discordant pairs is $Y$ and the total number of pairs is $N$, then the odds ratio is $(2 . X / Y) /(Y /(2 .(N-X-Y))$.

One note concerning possible confounding factors is in order. It would be desirable to control for effects of both age and gender in the calculation of sibling resemblance. However, in the present study, there is not sufficient power to stratify by gender composition or by age, the approach most appropriate when calculating pairwise odds ratios (Hunt $e$ t al., 1986). Hence, no attempts were made 
to examine the influences of age and gender on sibling resemblance in this paper. It should be noted, however, that the longitudinal design does address in part how age may affect the index of sibling resemblance.

\section{Results}

Sibling resemblance for major depression, anxiety disorder, and conduct disorder

Tables 2 and 3 present pairwise odds ratios for the three disorders of interest, at the two timepoints in the study (Waves 1 and 2), separately for offspring at high and low risk. In the offspring at high risk, sibling resemblance was significant for major depression and anxiety disorder at Wave 1, and was significant for major depression, anxiety disorder, and conduct disorder at Wave 2. An offspring at high risk was over twice as likely to have received a lifetime diagnosis of major depression if a sibling had a history of major depression at both Waves 1 and 2. An offspring at high risk was over twice as likely to have received a lifetime diagnosis of anxiety disorder if a sibling had a history of anxiety disorder at Wave 1 , and this probability ir.creased to almost three times as likely at Wave 2. Sibling resemblance for conduct disorder reached significance at Wave 2, as an offspring at high risk was over 2.5 times as likely to have received a lifetime diagnosis of conduct disorder if a sibling had a history of conduct disorder.

Table 2. Sibling resemblance for depression, anxiety disorder, and conduct disorder at Wave 1 in offspring at high and low risk

\begin{tabular}{lcccc}
\hline \multicolumn{5}{c}{$\%(N)$ of concordant and discordant sibling pairs* } \\
& Concordant & Discordant & Odds ratio & $\chi^{2}$ \\
\hline Depression & $\%(N)$ & $\%(N)$ & & \\
$\quad$ Low risk & $8.8 \%(6)$ & $30.9 \%(21)$ & 2.23 & 1.73 \\
$\quad$ High risk & $12.8 \%(21)$ & $34.1 \%(56)$ & 2.33 & $5.62^{*}$ \\
& & & & \\
Anxiety & & & 0.92 & 0.01 \\
$\quad$ Low risk & $2.9 \%(2)$ & $29.4 \%(20)$ & 2.16 & $5.27 \dagger$ \\
$\quad$ High risk & $17.1 \%(28)$ & $37.8 \%(62)$ & & \\
Conduct disorder & & & & \\
$\quad$ Low risk & $1.5 \%(1)$ & $19.1 \%(13)$ & 1.28 & 0.04 \\
$\quad$ High risk & $6.7 \%(11)$ & $35.9 \%(59)$ & 1.19 & 0.17 \\
\hline
\end{tabular}

*"Concordant" indicates both siblings in a sibling pair are affected; "Discordant" means one sibling in a sibling pair is affected.

$\dagger p<.05$. 
Table 3. Sibling resemblance for depression, anxiety disorder, and conduct disorder at Wave 2 in offspring at high and low risk

\begin{tabular}{lcccc}
\hline \multicolumn{4}{c}{$\%(N)$ of concordant and discordant sibling pairs* } \\
& Concordant & Discordant & Odds ratio & $\chi^{2}$ \\
\hline Depression & $\%(N)$ & $\%(N)$ & & \\
$\quad$ Low risk & $8.8 \%(6)$ & $30.9 \%(21)$ & 2.23 & 1.73 \\
$\quad$ High risk & $16.5 \%(27)$ & $38.4 \%(63)$ & 2.01 & $4.35 \dagger$ \\
Anxiety disorder & & & \\
$\quad$ Low risk & $2.9 \%(2)$ & $29.4 \%(20)$ & 0.92 & 0.01 \\
$\quad$ High risk & $23.1 \%(38)$ & $36.0 \%(59)$ & 2.93 & $10.85_{\ddagger}^{+}$ \\
Conduct disorder & & & & \\
$\quad$ Low risk & $4.4 \%(3)$ & $22.1 \%(15)$ & 2.67 & 1.64 \\
$\quad$ High risk & $15.8 \%(26)$ & $34.8 \%(57)$ & 2.59 & $7.71_{+}^{+}$ \\
\hline
\end{tabular}

* "Concordant" indicates both siblings in a sibling pair are affected; "Discordant" means one sibling in a sibling pair is affected.

$+p<.05$.

$\pm p<.01$.

The pairwise odds ratios for the three disorders of interest did not reach significance in the offspring at low risk at Waves 1 and 2. However, it should be noted that the pairwise odds ratios for major depression and conduct disorder in the offspring at low risk were of similar magnitude to those found for the offspring at high risk. The difference in significance levels were due in part to the smaller sample size of the offspring at low risk. Large differences in sibling resemblance between the offspring at high and low risk were apparent for anxiety disorder, and the difference in odds ratios between groups was significant $(p<.05)$ for the Wave 2 data. It should be noted that the pairwise odds ratios for depression and anxiety disorders in the offspring at low risk did not change from Waves 1 and 2 because no new incidences of the disorders under study were found in Wave 2 in the low risk sample (Warner et al., 1992).

\section{Sibling resemblance for comorbid disorders}

Tables 4 and 5 present pairwise odds ratios for the three comorbid conditions (anxiety/depression, anxiety/conduct disorder, and depression/conduct disorder, calculated at the two timepoints in the study separately for siblings at high and low risk. In the high risk sample, sibling resemblance for anxiety/depression was significant at both timepoints; sibling resemblance for anxiety/conduct disorder was significant in the high risk cohort at the second timepoint; and these pairwise odds ratios were significantly larger $(p<.05)$ than those obtained for the low risk cohort. 
Table 4. Sibling resemblance for comorbid conditions at Wave 1

\begin{tabular}{lcccc}
\hline \multicolumn{5}{c}{$\%(N)$ of concordant and discordant sibling pairs* } \\
& Concordant & Discordant & Odds ratio & $\chi^{2}$ \\
\hline Anxiety/Depression & $\%(N)$ & $\%(N)$ & \\
$\quad$ Low risk & $1.5 \%(1)$ & $19.1 \%(13)$ & $\dagger$ & \\
High risk & $7.3 \%(12)$ & $21.9 \%(36)$ & 4.30 & $11.91 \ddagger$ \\
& & & \\
Anxiety/conduct disorder & $0.0 \%(0)$ & $5.9 \%(4)$ & $\dagger$ \\
$\quad$ Low risk & $1.2 \%(2)$ & $15.2 \%(25)$ & $\dagger$ \\
High risk & & & \\
& & & \\
Conduct disorder/depression & $0.0 \%(0)$ & $16.2 \%(11)$ & \\
$\quad$ Low risk & $1.2 \%(2)$ & $23.2 \%(38)$ & $\dagger$ \\
High risk & & & \\
\hline
\end{tabular}

*"Concordant" indicates both siblings in a sibling pair are affected; "Discordant" means one sibling in a sibling pair is affected.

$\dagger$ Too few affected cases to calculate odds ratio.

$\pm p<.01$.

Table 5. Sibling resemblance for comorbid conditions at Wave 2

\begin{tabular}{|c|c|c|c|c|}
\hline \multicolumn{5}{|c|}{$\%(N)$ of concordant and discordant sibling pairs* } \\
\hline & Concordant & Discordant & Odds ratio & $\chi^{2}$ \\
\hline Anxiety/depression & $\%(N)$ & $\%(N)$ & & \\
\hline Low risk & $1.5 \%(1)$ & $19.1 \%(13)$ & $\dagger$ & \\
\hline High risk & $9.7 \%(16)$ & $25.6 \%(42)$ & 3.84 & $11.75_{+}^{+}$ \\
\hline \multicolumn{5}{|c|}{ Anxiety/conduct disorder } \\
\hline Low risk & $0.0 \%(0)$ & $5.9 \%(4)$ & $\dagger$ & \\
\hline High risk & $6.1 \%(10)$ & $14.0 \%(23)$ & 9.90 & $24.19 \ddagger$ \\
\hline \multicolumn{5}{|c|}{ Conduct disorder/depression } \\
\hline Low risk & $0.0 \%(0)$ & $16.2 \%(11)$ & + & \\
\hline High risk & $4.8 \%(8)$ & $25.0 \%(41)$ & 2.19 & 3.16 \\
\hline
\end{tabular}

* "Concordant" indicates both siblings in a sibling pair are affected; "Discordant" means one sibling in a sibling pair is affected.

†Too few affected cases to calculate odds ratio.

$\ddagger p<.01$.

\section{Discussion}

A surprising finding of this study was that the magnitude of sibling resemblance for depression was very similar for the high- and low risk cohorts. This suggests that the common or shared familial influences which impact the development of depression in multiple children in a family are not more "powerful" in families at high risk via parental depression. We hypothesize that the similar magnitude of sibling resemblance across cohorts implies that there are some common pathways 
to depression in offspring at high and low risk, perhaps involving well-studied environmental influences such as family discord (e.g. Rutter, 1990). Such a hypothesis is also consistent with the similar magnitude of sibling resemblance for conduct disorder in both cohorts observed in this study. Taken together, these findings suggest that the common or shared influences on siblings at either high or low risk for depression may exert a general effect on a wide range of psychopathology, including both depression and conduct disorder.

The notable distinction between the high and low risk cohorts, in terms of sibling resemblance, was found for anxiety disorders, rather than depression. This finding is consistent with speculations that early expression of anxiety could reflect an underlying vulnerability to emotional disorders transmitted by depressed parents by either genetic or shared environmental factors (Merikangas, 1993). Hence, examinations of siblings resemblance in this study suggest that attempts to specify familial factors-including genetic influences-representing a liability to affective disorders in offspring at high risk focus on anxiety disorders, rather than depression.

Similarly, the focus on comorbid conditions revealed that sibling resemblance for the comorbid condition anxiety disorder/depression was significant for the high risk sample at both timepoints. The evidence for sibling resemblance for anxiety disorder/depression is consistent with research indicating that major depression and anxiety disorder may be due to common genetic influences (Kendler et al., 1992). In addition, at the second timepoint, sibling resemblance for anxiety disorder/conduct disorder also was significant, whereas sibling resemblance for conduct disorder/major depression was not. These results may be interpreted to mean that familial influences common to siblings may be an important etiological factor in the development of psychiatric disorders which aggregate with anxiety disorder within individuals, including depression. Again, this speculation is consistent with Merikangas' (1993) assertion that familial aggregation for depression may operate via anxiety, rather than depression per se.

Overall, the magnitude of sibling resemblance for psychiatric disorders in the offspring at high and low risk should be described as a moderate effect size (with the possible exception of sibling resemblance for anxiety disorders in the high risk cohort). This finding is consistent with research suggesting that sibling resemblance is often modest, even though siblings share the same parents and a common genetic background (e.g. Dunn \& Plomin, 1990). The results of this paper may be interpreted to mean that etiological factors which operate to produce dissimilarities between siblings at high risk for psychiatric disorders are not trivial and should be considered in future research along with familial influences which impinge similarly on siblings at risk for psychiatric disorders (Reiss et al., 1991; Rende \& Plomin, 1993; Rutter et al., 1990). One caveat is in order, namely that the potential effects of age and gender on sibling resemblance could not be analyzed in this study because of severe power limitations. Hence, future studies should determine if sibling resemblance is conditional on age of offspring, age gap between siblings, and gender composition of the sibling pairs.

There was little evidence for changes in sibling resemblance in the high risk cohort from the initial assessment period and the 2-year follow-up, although there is some evidence that sibling resemblance for comorbid conditions increased over 
the 2-year period. One possibility is that the 2-year time period is insufficient to observe drastic changes in sibling resemblance. The high and low risk cohorts are currently being assessed in a 10-year follow-up study, at which point nearly all of the subjects have entered early adulthood. Examination of sibling resemblance in the 10-year follow-up will provide a better test of the hypothesis that sibling resemblance for psychiatric disorders in offspring at high risk increases with age (or as most offspring pass through the age of first onset of disorders).

Two directions for future studies should be noted. First, sibling resemblance in offspring at high and low risk should be examined once most of the offspring pass through the age of first onset for the disorders under study in order to generate more definitive numbers of sibling resemblance for history of psychiatric disorders. As mentioned earlier, the ten-year follow-up of the cohorts participating in this study will allow this goal to be met. Second, there should be a focus on identifying risk factors which contribute to sibling resemblance for psychiatric disorders, as well as risk factors which impinge differently on offspring in the same family. We plan to address these issues in a future paper.

Acknowledgements-This research was supported in part by the National Institute of Mental Health Grants \#MH36197 and \#MH28274 (Dr. Weissman), grant \#86-213 from the John D. and Catherine T. MacArthur Foundation Mental Health Research Network on Risk and Protective Factors in the Major Mental Disorders (Dr. Weissman), a NARSAD Young Investigator Award (Dr. Rende), and an Aaron Diamond Foundation Postdoctoral Fellowship in the Biomedical and Social Sciences (Dr. Rende). We thank Hsiaoyun Hsu for assistance with statistical analyses and programming.

\section{References}

American Psychiatric Association (1987). Diagnostic and statistical manual of mental disorders (DSM-III-R) (3rd ed. revised). Washington, DC: American Psychiatric Association.

Caron, C. \& Rutter, M. (1991). Comorbidity in child psychopathology: concepts, issues, and research strategies. Journal of Child Psychology and Psychiatry, 32, 1063-1080.

Dunn, J. \& Plomin, R. (1990). Separate lives: why siblings are so different. New York: Basic Books.

Hammen, C., Burge, D., Burney, E. \& Adrian, C. (1990). Longitudinal study of diagnoses in children of women with unipolar and bipolar affective disorder. Archives of General Psychiatry, 47, 1112-1117.

Hammen, C., Gordon, D., Burge, D., Adrian, C., Jaenicke, C. \& Hiroto, D. (1987). Maternal affective disorder, illness and stress: risk for children's psychopathology. American Jourmal of Psychiatry, 144, 736-741.

Hunt, S. C., Hasstedt, S. J. \& Williams, R. R. (1986). Testing for familial aggregation of a dichotomous trait. Genetic Epidemiology, 3, 299-312.

Kendler, K. S., Neale, M. C., Kessler, R. C., Heath, A. C. \& Eaves, L. J. (1992). Major depression and generalized anxiety disorder: same genes, (partly) different environments? Archives of General Psychiatry, 49, 716-722.

Khoury, M. J., Beaty, T. H. \& Cohen, B. H. (1993). Fundamentals of genetic epidemiology: monographs in epidemiology and biostatistics (Vol. 19). New York: Oxford University Press.

Leckman, J. F., Sholomskas, D., Thompson, W. D., Belanger, A. \& Weissman, M. M. (1982). Best estimate of lifetime psychiatric diagnosis, Archives of General Psychiatry, 39, 879-883.

Merikangas, K. R. (1993). Genetic epidemiological studies of affective disorders in childhood and adolescence. European Archives of Psychiatry and Clinical Neuroscience, 243, 121-130.

McGue, M. (1991). When assessing twin concordance, use the probandwise not the pairwise rate. Schizophrenia Bulletin, 18, 171-176. 
Orvaschel, H., Puig-Antich, P., Chambers, W., Tabrizi, M. A. \& Johnson, R. (1981). Retrospective assessment of prepubertal major depression with the Kiddie-SADS-E. Joumal of the American Academy of Child and Adolescent Psychiatry, 38, 392-397.

Reiss, D., Plomin, R. \& Hetherington, E. M. (1991). Genetics and psychiatry: an unheralded window on the environment. American Journal of Psychiatry, 148, 283-291.

Rende, R. \& Plomin, R. (1993). Families at risk for psychopathology: who becomes affected and why? Development and Psychopathology, 5, 529-540.

Rende, R. \& Weissman, M. M. (in press). Assessment of family history of psychiatric disorder. In D. Shaffer \& J. Richters (Eds), Assessment of child psychopathology. New York: Guilford.

Rutter, M. (1990). Commentary: some focus and process considerations re the effects on children of parental depression. Developmental Psychology, 26, 60-63.

Rutter, M., Bolton, P., Harrington, R., Le Couteur, A., Macdonald, H. \& Simonoff, E. (1990). Genetic factors in child psychiatric disorders-I. A review of research strategies. Journal of Child Psychology and Psychiatry, 31, 3-37.

Warner, V., Weissman, M. M., Fendrich, M., Wickramaratne, P. \& Moreau, D. (1992). The course of major depression in the offspring of depressed parents: incidence, recurrence, and recovery. Archives of General Psychiatry, 49, 795-801.

Weissman, M. M., Fendrich, M., Warner, V. \& Wickramaratne, P. (1991). Incidence of psychiatric disorder in offspring at high and low risk for depression. Jourmal of the American Academy of Child and Adolescent Psychiatry, 31, 640-648.

Weissman, M. M., Gammon, G. D., John, K., Merikangas, K. R., Warner, V., Prusoff, B. A. \& Sholomskas, D. (1987). Children of depressed parents: increased psychopathology and early onset of major depression. Archives of General Psychiatry, 44, 847-853.

Weissman, M. M., Merikangas, K. R., John, K., Wickramaratne, P., Prusoff, B. A. \& Kidd, K. K. (1986). Family-genetic studies of psychiatric disorders. Archives of General Psychiatry, 43, 1104-1116.

Wickramaratne, P. J., Caron, C., Warner, V. \& Weissman, M. M. (submitted). The joint effects of family risk factors on depression in offspring at high and low risk for depression. 
This document is a scanned copy of a printed document. No warranty is given about the accuracy of the copy. Users should refer to the original published version of the material. 